\title{
Reduced ethylene production in tomato fruits upon CRISPR/Cas9-mediated LeMADS-RIN mutagenesis
}

\author{
Jung Yu Jin ${ }^{1}$, Lee Geung-Joo ${ }^{2}$, Bae Sangsu ${ }^{3}$, Kang Kwon Kyoo ${ }^{1 *}$ \\ ${ }^{1}$ Department of Horticultural Life Science, Hankyong National University, Ansung, Korea \\ ${ }^{2}$ Department of Horticulture, Chungnam National University, Daejeon, Korea \\ ${ }^{3}$ Department of Chemistry, Hanyang University, Seoul, Korea \\ *e-mail:kykang@hknu.ac.kr
}

Recent progress in genome editing methods has opened new opportunities for reverse genetics-based studies in plants. The clustered regularly interspaced short palindromic repeat (CRISPR) system is a novel strategy used to induce mutations in a specific genomic region of a variety of organisms, including plants. Here, we describe a high-frequency targeted mutagenesis utilizing Agrobacterium-delivered CRISPR/Cas9 in tomato. This system consists of an Agrobacterium binary vector and three guide RNAs for single gene targeting. We evaluated the system for its mutagenesis frequency and heritability using LeMADS-RIN gene of tomato. $\mathrm{T}_{0}$ transgenic events carrying mutations in the LeMADS-RIN gene occurred at rates over $10.6 \%$ mutants per transgenic event in both 'Mamirio' and 'Golden bell' tomato genotypes. Three independent $\mathrm{T}_{1}$ transgenic lines and wild-type (WT) tomato plants were used for ethylene analysis. Compared with WT plants, edited mutants exhibited more incompletely-ripening fruits and lower ethylene contents. Following genetic combination through segregation, null segregants carrying only the desired mutant alleles without the CRISPR transgene could be retrieved among the $\mathrm{T}_{1}$ progeny. These Cas9/gRNA transgenic lines, therefore, can be used to convey the CRISPR-based mutagenesis by genetic cross to tomato lines that are not amenable to genetic transformation.

Acknowledgements: This work was supported by a grant from the Next-Generation BioGreen 21 Program (PJ01319302), RDA and Golden Seed project (213007-05-2SBD30), Republic of Korea. 